\title{
A Method of Initial Velocity Measurement for Rocket Projectile
}

\author{
Jiancheng Zhang ${ }^{1}$, Tianle Zhang ${ }^{2}$, Zhengyu $\mathrm{Cai}^{3}$ \\ ${ }^{1}$ School of Electronic and Optical Engineering, Nanjing University of Science and Technology, 210094, Nanjing, China \\ ${ }^{2}$ School of Electronic and Optical Engineering, Nanjing University of Science and Technology, 210094, Nanjing, China \\ ${ }^{3}$ School of Electronic and Optical Engineering, Nanjing University of Science and Technology, 210094, Nanjing, China
}

\begin{abstract}
In this paper, a novel method is proposed to measure the initial velocity of the rocket based on STFT (the short-time Fourier transform) and the WT (wavelet transform). The radar echo signal processing procedure involves the following steps: sampling process, overlapping windows, wavelet decomposition and reconstruction, computing FFT (Fast Fourier Transform) and spectrum analysis, power spectrum peak detection. Then, according to the peak of the detection power spectrum, the corresponding Doppler frequency is obtained. Finally, on the basis of the relationship between Doppler frequency and instantaneous velocity, the V-T curve is drawn in MATLAB to obtain the initial velocity of the rocket muzzle.
\end{abstract}

\section{Introduction}

With the development of weapon system, people have higher requirements on the performance of weapon system. As an important indicator of weapon characteristics and trajectory characteristics, the velocity and acceleration of ammunition are widely used in the research of weapon system. Therefore, the initial velocity of muzzle is of great significance to the development of weapon system and the development of ballistics theory. In this paper, we present a new method for initial velocity measurement by combining STFT with wavelet transform.

\section{Radar echo signal model}

The radar echo signal of the rocket is usually considered as stationary random signal in a short time and the instantaneous Doppler frequency is time-varying [1]. Thence the echo signal model of the rocket can be expressed as formula (1).

$$
x(t)=\sum_{i=1}^{n} a_{i} \sin \left(2 \pi f_{i} t+\varphi\right)+\sum_{i=1}^{n} \rho_{\omega} \delta(i)
$$

Where $f_{i}$ is the frequency of the $i^{\text {th }}$ signal; $a_{i}$ is the amplitude of the $i^{\text {th }}$ signal; $\rho_{\omega} \delta(i)$ is the $i^{\text {th }}$ noise signal.

Therefore, the core of signal processing is how to extract the Doppler frequency that the radial velocity corresponds to from the radar echo signal of the rocket. In order to extract the time-varying characteristic of echo signal, we take advantage of the method of the timefrequency analysis to describe the echo signal of the rocket. Time-frequency analysis methods primarily include the following sections: the short-time Fourier transform, wavelet transform, WVD (wigner-ville distribution) transformation, fractional Fourier transform, polynomial phase transformation, Dechirp transform method, and so on [2]. This paper aims at proposing a new design method for processing radar signal that combine STFT with wavelet transform. By overlaying the window with the advantage of high magnitudes of the Blackman window in the time domain, it is possible to extract useful information that is missed at the edge of the window.

\section{Radar echo signal analysis}

\subsection{The short-time Fourier transform}

In practice, the procedure for computing STFT is to divide a longer time signal into shorter segments of equal length and then compute the Fourier transform separately on each shorter segment [3]. Then we usually plot the changing spectra as a function of time.

In the discrete time case, the data to be transformed could be broken up into chunks or frames (which usually overlap each other, to reduce artifacts at the boundary). Each chunk is Fourier transformed, and the complex result is added to a matrix, which records magnitude and phase for each point in time and frequency. The formula can be delivered as

$$
X(m, \omega)=\sum_{n=-\infty}^{\infty} x[n] w[n-m] e^{-j \omega n}
$$

In formula (2), where $\mathrm{x}[n]$ is the signal function, $\mathrm{w}[n]$ is the window function, $m$ and $\omega$ are discrete and quantized respectively. $\mathrm{X}(m, \omega)$ is the Fourier Transform of $x[n] w[n-m]$.

$\overline{{ }^{1} \text { Corresponding author: jiancheng_z@ } 163 . c o m}$ 


\subsection{Wavelet de-noising}

Wavelet transform is a new transform analysis method, which inherits and develops the idea of localized STFT, and overcomes the shortcomings of window size not changing with frequency. It is an ideal tool for signal timefrequency analysis and processing [4]. In the continuous time case, continuous wavelet transform can be expressed as

$$
W_{\varphi} f(a, b)=\frac{1}{\sqrt{|a|}} \int_{-\infty}^{+\infty} f(t) \varphi^{*}\left(\frac{t-b}{a}\right) d t
$$

Where $a(a \in R, a \neq 0)$ is scale factor and denotes the stretch and shrink of the frequency, $b$ is time shift factor.

$$
\varphi_{a, b}(t)=\frac{1}{\sqrt{|a|}} \varphi\left(\frac{t-b}{a}\right)
$$

The above-mentioned formula (4) is wavelet mother function.

In our design, wavelet transform is utilized to remove part of the noise signal.

\subsection{Power spectrum estimation algorithm}

In a short time, the radar echo signal of the rocket belongs to stationary random signal, therefore we adopt the method of power spectrum estimation in signal processing [5]. This power spectrum estimation algorithm is based on the Fourier Transform, and there are two methods to realize this algorithm. The detailed theoretical analyses would be showed as follows.

During signal processing, the periodogram is an estimate of the spectral density of a signal [6]. Assume that the random signal is $x(n)$, we intercept $\mathrm{N}$ points of $x(n)$ and it is denoted as $x_{N}(n)$. Then computing DFT (Discrete Fourier Transform) for $x_{N}(n)$ and its corresponding spectrum is expressed as $X_{N}(k)$.

$$
X_{N}(k)=\sum_{n=0}^{N-1} x_{N}(n) e^{-j \omega k n}
$$

Where $\omega=\frac{2 \pi}{N}, n=0,1,2 \ldots . N-1$. For discrete signals, the power spectrum estimation value is expressed as

$$
\hat{P}_{P E R}(k)=\frac{1}{N}\left|X_{N}(k)\right|^{2}
$$

The other method is the autocorrelation power spectrum estimation. We can obtain the autocorrelation function for $x_{N}(n)$, and it is expressed as

$$
\hat{r}(m)=\frac{1}{N} \sum_{n=0}^{N-1} x(n+k) x^{*}(n), k=0,1,2, \ldots, M
$$

The power spectrum estimation is expressed as

$$
\hat{P}_{B T}(\omega)=\sum_{m=-M}^{M} \hat{r}(m) e^{-j \omega m},|M| \leq N-1
$$

When $M<<-1, \quad \hat{P}_{B T}(\omega)$ makes the signal smooth and improves the method of the periodogram. When $M=N-1$, the periodogram is a special case of the autocorrelation power spectrum estimation.

\section{ALGORITHM DESCRITION}

\subsection{Signal processing process}

The data used in this paper are collected by the Doppler velocimetry radar of the $35 \mathrm{GHz}$ continuous wave system and the sampling frequency is $0.25 \mathrm{MHz}$. The process of signal processing involves the following steps:

(1) The discrete radar echo signal of the rocket are divided into 200 segments and the length of each segment is 1024 .

(2) According to the Mallat algorithm, the db12 wavelet is selected to filter each segment of the signal, then utilize the method of the tower decomposition and reconstruction to obtain the approximate component of the signal [7].

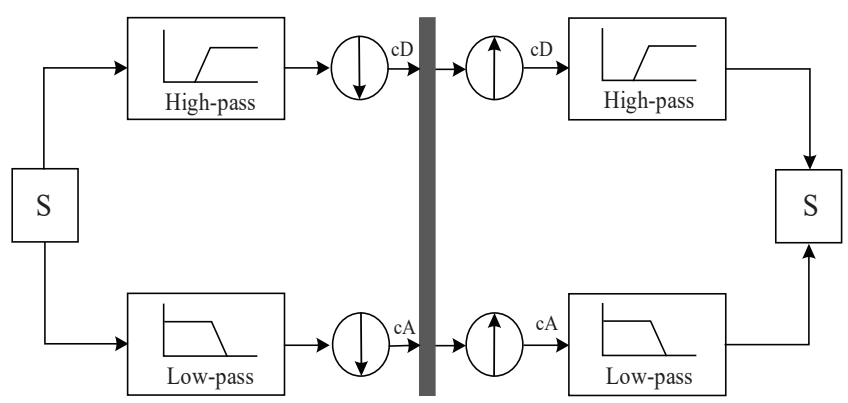

Figure 1. Realization of the Mallat algorithm.

Figure 1 shows the procedure of the whole decomposition and reconstruction. But in our paper, we just need to gain low frequency wavelet coefficient. That would be explained detailly in Figure 2.

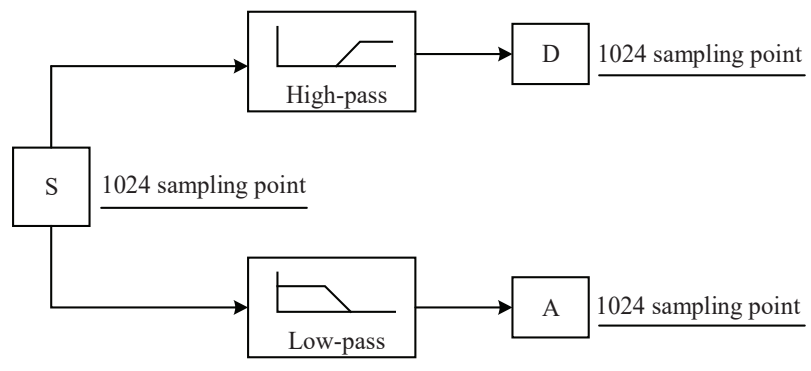

Figure 2. Obtaining the low frequency wavelet coefficient.

The db12 wavelet is selected to compute DWT (Discrete Wavelet Transform) for the approximate component of the signal, then we take the method of wavelet threshold denoising to process low frequency wavelet coefficient, which is depicted as cA in Figure 1. The $\mathrm{db} 12$ wavelet is a kind of Daubechies wavelet, it is used to decompose and refactor signals or as a filter [8]. It would be showed in detail in Figure 3. 

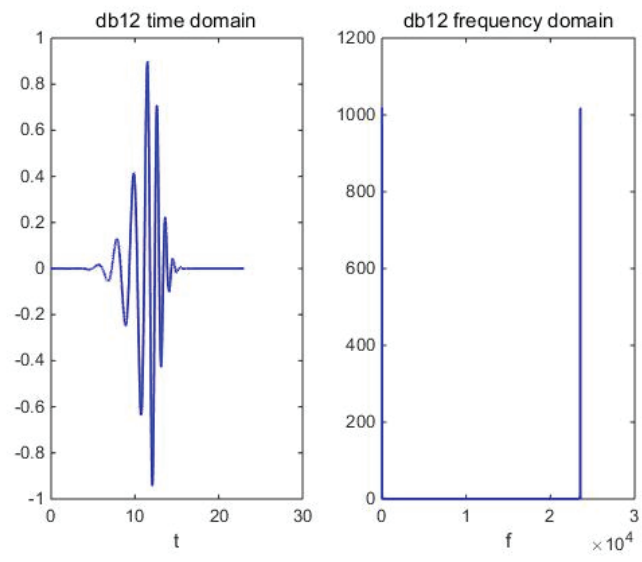

Figure 3. Db12 wavelet.

(3) Each segment of the signal computes 1024 point FFT transformation, and detecting the peak value of the power spectrum to obtain corresponding Doppler frequency and instantaneous velocity. Moving the sliding window back 512 points and circular 200 times. Finally, a power spectrum waterfall diagram that contains 200 groups signal is drawn in MATLAB. That would be showed in detail in Figure 4.

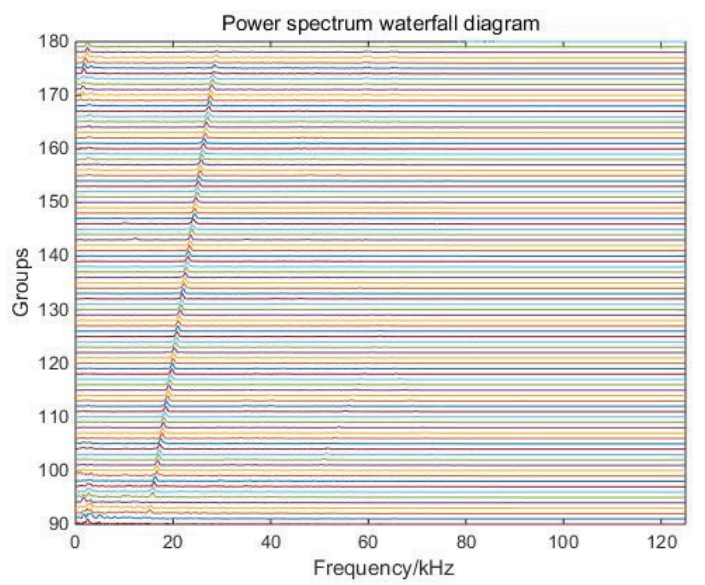

Figure 4. Power spectrum waterfall diagram.

(4) In this paper, the frequency of the radar transmit signal is $35 \mathrm{GHz}$, it is expressed as $f_{1}$. The sampling frequency is $0.25 \mathrm{MHz}$, it is expressed as $f_{s}$. For discrete signals, the actual frequency is expressed as

$$
f=\frac{n f_{s}}{N}
$$

Where $n$ is the position of the sampling point; $\mathrm{N}$ is the length of a window, in this paper, the length of a window is 1024 . The instantaneous velocity can be computed by the classical equation:

$$
v=\frac{\lambda f_{d}}{2}
$$

Where $f_{d}=\frac{d f_{s}}{N}, \lambda=\frac{C}{f_{1}}$.

The involved symbols in (10) are explained as follow $f_{\mathrm{d}}$ : the Doppler frequency,

$d$ : the position of the sampling point that the peak of the power spectrum corresponding, $\lambda$ : the wavelength of the radar transmit signal in free space,

$c$ : the speed of the electromagnetic wave in free space.

According to the relationship between the Doppler frequency and instantaneous velocity of the rocket, we fitting V-T curve in MATLAB by using the method of least squares. It is showed in Figure 5.

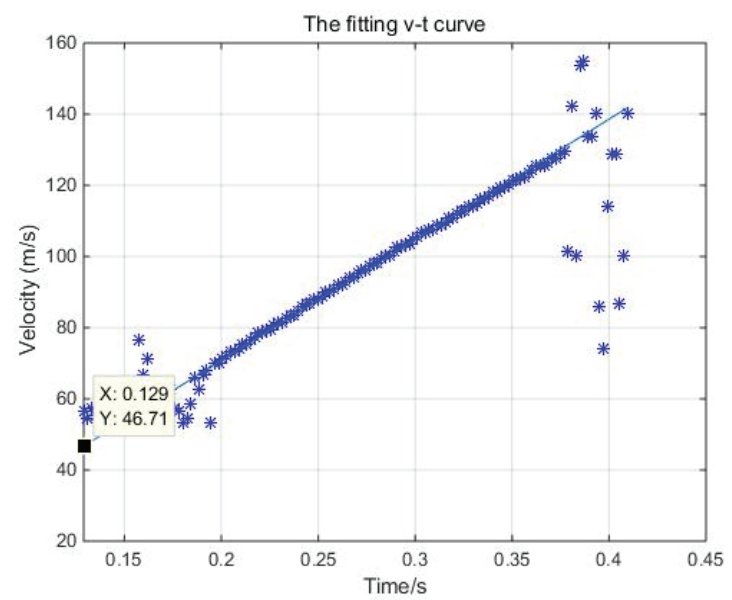

Figure 5. The fitting V-T curve.

Finally, we briefly show the process of signal processing with a flow chart in MATLAB. That is showed in detail in Figure 6.

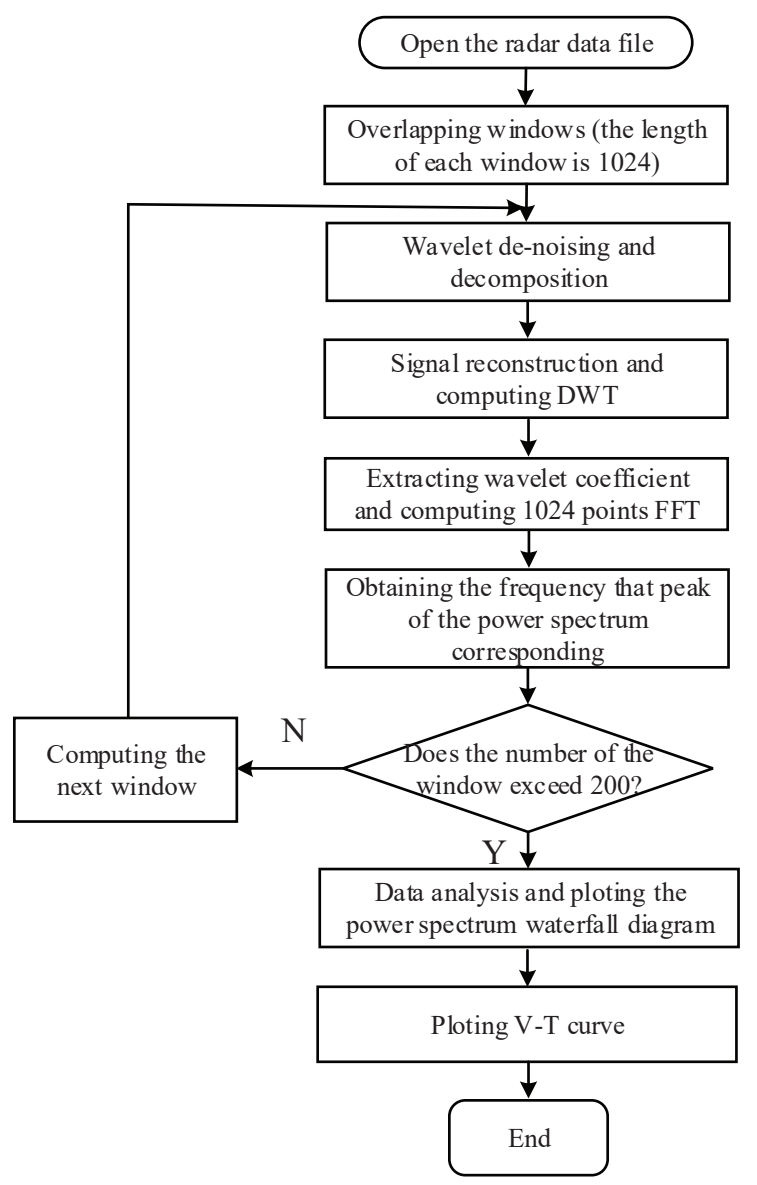

Figure 6. The flow chart of the signal processing 


\subsection{Data analysis}

We can obtain the instantaneous velocity of the rocket from the Figure 5, the data would be showed in detail in Table 1.

Table 1. Initial velocity fitting data.

\begin{tabular}{|c|c|c|c|c|c|c|}
\hline Time/s & 0.129 & 0.131 & 0.133 & 0.135 & 0.137 & 0.139 \\
\hline Velocity(m/s) & 46.71 & 47.99 & 48.71 & 49.41 & 50.11 & 50.82 \\
\hline Time/s & 0.141 & 0.143 & 0.145 & 0.147 & 0.149 & 0.152 \\
\hline Velocity(m/s) & 51.51 & 52.21 & 52.91 & 53.61 & 54.32 & 55.02 \\
\hline Time/s & 0.154 & 0.156 & 0.158 & 0.160 & 0.162 & 0.164 \\
\hline Velocity(m/s) & 55.72 & 56.43 & 57.12 & 57.83 & 58.53 & 59.23 \\
\hline Time/s & 0.166 & 0.168 & 0.169 & 0.172 & 0.174 & 0.176 \\
\hline Velocity(m/s) & 59.93 & 60.63 & 61.34 & 62.04 & 62.74 & 63.44 \\
\hline
\end{tabular}

From the above table, it shows that the instantaneous velocity of the rocket is $46.71 \mathrm{~m} / \mathrm{s}$ when the time is $129 \mathrm{~ms}$ and $47.99 \mathrm{~m} / \mathrm{s}$ when time is $131 \mathrm{~ms}$. And on the basis of the muzzle vibration test, the time that this rocket went out of the muzzle is $130 \mathrm{~ms}$. Finally, in order to get the instantaneous velocity when time is $130 \mathrm{~ms}$, we take the average of these two instantaneous velocity value. The result shows that the instantaneous velocity of the rocket is $47.35 \mathrm{~m} / \mathrm{s}$ when the time is $130 \mathrm{~ms}$. Therefore, we can draw a conclusion that the initial velocity of the rocket is $47.35 \mathrm{~m} / \mathrm{s}$ when this rocket went out of the muzzle.

\section{Conclusion}

A novel method of initial velocity measurement of the rocket is proposed in this paper. This algorithm has a low calculation and is more suitable for the radar signal. The result of the actual signal processing verify that the proposed method achieves better estimation accuracy than the traditional spectrum analysis, and the segment of useful information that related to the target can be extracted effectively in the low SNR (Signal Noise Ratio) conditions.

\section{Acknowledgment}

The authors would like to thank the members of Radar Signal Processing Laboratory of Nanjing University of Science and Technology for their help to this paper.

\section{References}

1. J. Flamant; N. Le Bihan; P. Chainais, "Spectral analysis of stationary random bivariate signals," in IEEE Transactions on Signal Processing, vol.PP, no.99, pp.1-1.

2. B. Wu, K. Li, M. Yang and C. H. Lee, "A study on sampling of STFT modifications in time and frequency domains for DNN-based speech dereverberation,"2016 Asia-Pacific Signal and Information Processing Association Annual Summit and Conference (APSIPA), Jeju, 2016, pp. 1-4.
3. F. Qifeng, C. Guoqing and S. Zibo, "Application of wavelet de-noising method in vibration signal analysis of elevator car," 2016 13th International Conference on Ubiquitous Robots and Ambient Intelligence (URAI), Xi'an, 2016, pp. 610-614.

4. Y. Zhao, L. Zhang, J. Hu and T. Liao, "Mallat Wavelet Filter Coefficient Calculation," 2013 International Conference on Computational and Information Sciences, Shiyang, 2013, pp. 963-965.

5. P. Balakrishnan, M. M. Hasan and K. A. Wahid, "An Efficient Algorithm for Daubechies Lifting Wavelets Using Algebraic Integers," in Canadian Journal of Electrical and Computer Engineering, vol. 37, no. 3, pp. 127-134, Summer 2014.

6. Zheng Cheng gong, Chen Xi and Huang Zhen, "A comprehensive analysis on Doppler frequency and Doppler frequency rate characterization for GNSS receivers," 2016 2nd IEEE International Conference on Computer and Communications (ICCC), Chengdu, 2016, pp. 2606-2610.

7. Y. Zhao, L. Zhang, J. Hu and T. Liao, "Mallat Wavelet Filter Coefficient Calculation," 2013 International Conference on Computational and Information Sciences, Shiyang, 2013, pp. 963-965.

8. P. Balakrishnan, M. M. Hasan and K. A. Wahid, "An Efficient Algorithm for Daubechies Lifting Wavelets Using Algebraic Integers," in Canadian Journal of Electrical and Computer Engineering, vol. 37, no. 3, pp. 127-134, Summer 2014. 